\title{
Leading Edge Aerothermal Inverse Design of Hyersonic Vehicle Based on Homotopy Optimization Method
}

\author{
K. Cui*, S. C. Hu, T. Y. Gao, X. P. Wang, G. W. Yang \\ LHD of Institute of Mechanics, Chinese Academy of Sciences, Beijing 100190, China
}

Email:kcui@imech.ac.cn

\begin{abstract}
Blunt leading edge with profiles of circular or power law shape is often used to decrease the aerodynamic heating of a vehicle when it flights into hypersonic regime. In order to further reduce the peak of heat flux of the leading edge, an inverse shape design method is presented in this paper. The leading edge is parameterized by using $B$-spline curve method. The hypersonic flow field and the heat flux distribution around the leading edge is evaluated by computational fluid dynamics. A homotopy method is developed as the optimizer. The computational heat flux distribution is driven by the optimizer to meet the objective. In order to verify the validity of the method, the inverse aerothermal design of a 2D leading edge with the thickness of $5 \mathrm{~mm}$ was carried out in the design condition Mach number is 6.5. The initial profile of the leading edge approximates to a circular arc. An H-type structured grid was used to discrete the computational domain. A 2D thin-layer Reynolds-averaged Navier-Stokes equations in strong conservation law form was employed as the solver. The results have shown that the peak value of the heat flux decreases about $4.6 \%$.
\end{abstract}

Key words: hypersonic, blunt leading edge, aerothermodynamic, inverse design, homotopy

\section{INTRODUCTION}

The hypersonic flight vehicles and its relative technologies have attracted lots of attention of many scientists and engineers. A successful design of hypersonic configuration will depend on the ability to incorporate relatively sharp leading edges that combine good aerodynamic properties with acceptable heating rates. As aerodynamic heating may cause serious problems at these speeds, the vehicle leading edges must be sufficiently blunt in order to reduce the heat transfer rate as much as possible, at least to acceptable levels for internal heat conduction [1]. The blunt leading edge is widely used to alleviate the aerodynamic heating problem since the heat flux for blunt bodies scales inversely with the square root of the nose radius. Traditionally, curves with analytical expression, such as circular arc shapes [2,3], power law shapes [4] are used as the the profile of then leading edge. Recently, the nose tip ablation method to reduce the severe heating around the nose of a hypersonic vehicle is studied [5].

Theoretical formulations, experimental data, and semi-empirical formulas all agree in the fact that stagnation point heat transfer for blunt body in continuum flow is inversely proportional to the square root of the nose radius of the leading edge [6], i.e.

$q \propto 1 / \sqrt{R_{\mathrm{n}}}$

here, $q$ denotes the heat flux and $R_{\mathrm{n}}$ denotes the nose radius. Obviously, the stagnation point heat flux is dependent on the shape of the leading edge. Hypersonic vehicle designers always expect the peak value of the heat flux decreases as much as possible to make the thermal protection system (TPS) become more reliable. Can we find a more appropriate shape to futher reduce the severe heating of the leading edge expect the circular shape and the power law shape metioned above? Naturelly, this question motives our study. In this paper, a inverse design methodology [7] is employed to obtain 
the optimal two dimensional profile of the leading edge. The 2D shape is parameterized by the $B$-spline method. A computational fluid dynamic (CFD)-based homotopy method is used as the optimizer. Numerical examples show that the peak value of the optimal shape decreases about $4 \%$ in comparison with the initial circular arc shape.

\section{PROCEDURE AND ALGORITHM OF INVERSE DESIGN}

The flowchart of the inverse design can be briefly summarized as follows. To begin with,the heating flux distribution of the initial circular arc shape with radius $2.5 \mathrm{~mm}$ is evaluated by CFD module based on structured grids. A supposed target distribution is given to reduce the peak value of the heating flux. Next, the shape is parameterized by a $B$-spline curve method. Thus the initial value of the design variables can be obtained. The initial shape and the target distribution are imported to the optimizer. The iteration driven by the optimizer will not be terminated until the target distribution is met or the optimal solution is found.

A homotopy-based continuation method is used as the driver of the optimization [8]. Homotopy is an important concept of topology originally. The discrete form of tht inversion problem is generally formulated to the following nonlinear optimization problem

$\min J(p)=\frac{1}{2}\left\|\boldsymbol{C}(\boldsymbol{p})-\boldsymbol{C}_{\mathrm{E}}\right\|^{2}$

where $\boldsymbol{C}(\boldsymbol{p})$ and $\boldsymbol{C}_{\mathrm{E}}$ are the computational and the target heating flux distribution respectively, $\boldsymbol{p}$ denote design variables vector. By apply the homotopy mapping, the following iteration scheme can be derived

$$
\begin{aligned}
& {\left[\left(1-\lambda^{n}\right) \boldsymbol{G}^{\mathrm{T}} \boldsymbol{G}+\lambda^{n} \boldsymbol{I}\right] \Delta \boldsymbol{p}=\left(1-\lambda^{n}\right) \boldsymbol{G}^{\mathrm{T}}\left(\boldsymbol{C}^{n}-\boldsymbol{C}_{\mathrm{E}}\right),} \\
& \boldsymbol{p}^{n+1}=\boldsymbol{p}^{n}+\Delta \boldsymbol{p},
\end{aligned}
$$

where $\lambda^{n}$ is the homotopy parameter. In order to ensure a stable iteration process, $\lambda$ should be diminished along a smooth path during iteration. Second, for restraining the measurement errors, $\lambda$ should be terminated at a proper point, which may close to zero, but should be unequal to zero. Here we propose a function, which is derived from the sigmoid function of the neural networks, as follows

$\lambda^{n}=\frac{1}{1+\mathrm{e}^{\beta\left(n-N_{0}\right)}}$,

where $n$ is the number of times of iteration, $\beta$ and $N_{0}$ are the adjustable parameters. It is clear that this function we call it the quasi-sigmoid function could naturally meet the two requirements mentioned above. Parameter $N_{0}$ is mainly used to ensure the stability of computation at the early stage of iteration, while $\beta$ can balance the stability and the efficiency of computation. The lower value of $N_{0}$ and $\beta$ can accelerate iteration, but may lead to a divergent process. In our problems, we suggest that $N_{0}$ should be an integer from 0 to 5 , and $\beta$ should be chosen from 0.01 to 0.5 .

\section{NUMERICAL RESULTS}

The computational conditions are as follows: the Mach number is 6.5, angle of attack is $0^{\circ}$, static pressure $1847.46(\mathrm{~Pa})$, static temperature is $223.65(\mathrm{~K})$, wall temperature is $300(\mathrm{~K})$. The standard $K-E$ model is adopted.

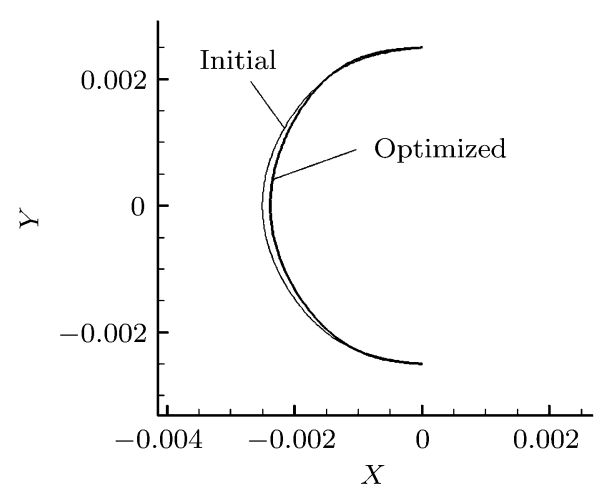

(a) Comparison of shapes

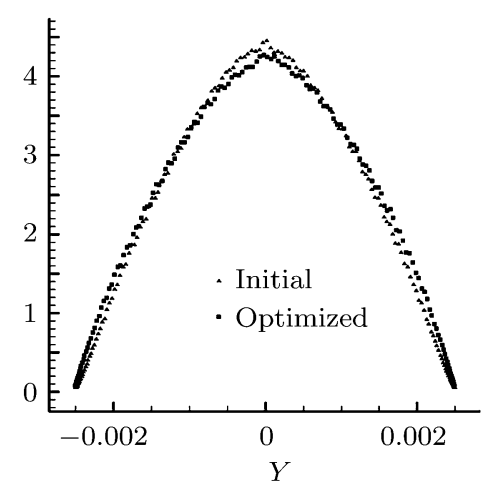

(b) Comparison of heating flux

Figure 1: Comparison between the initial and optimized shapes (a) and heating flux (b). 


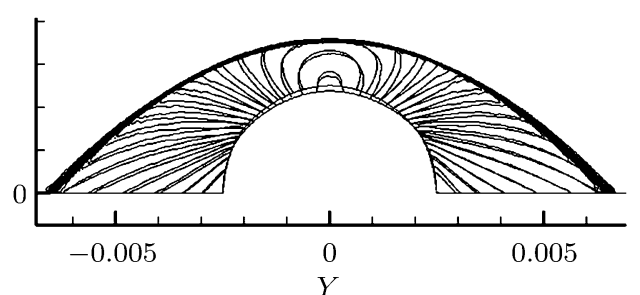

(a) Pressure countours comparision

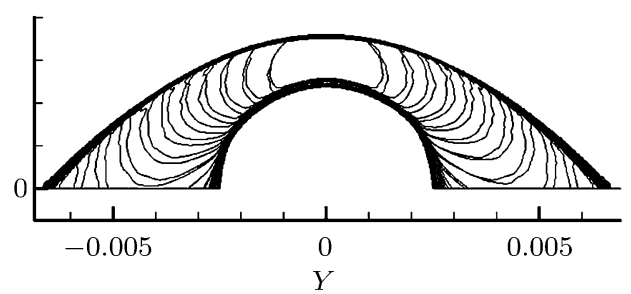

(b) Temperature countours comparision

Figure 2: Pressure (a) and temperature (b) countours comparision

Numerical resuts are shown in Figures 1 and 2, and Table 1. The peak value of the heating flux decreases about 4.6\%. The results clearly demonstrate the vadility of the optimization.

Table 1. Comparision of the control points and peak values of heating flux

\begin{tabular}{ccc}
\hline & Initial & Optimized \\
\hline & 0 & 0 \\
& -1.5882 & -1.4134 \\
Control points at $x$-axis $(\mathrm{mm})$ & -2.9638 & -2.8273 \\
& -1.6319 & -1.5571 \\
& 0 & 0 \\
\hline Control points at $y$-axis $(\mathrm{mm})$ & -2.5 & -2.5 \\
& -2.3226 & -2.3877 \\
& -0.03751 & -0.1085 \\
& 2.3095 & 2.395 \\
& 2.5 & 2.5 \\
\hline Peak values of heating flux $\left(\mathrm{W} / \mathrm{m}^{2}\right)$ & 4384059 & 4183844 \\
\hline Drag $(\mathrm{N})$ & 502.5 & 516.1 \\
\hline
\end{tabular}

\section{CONCLUSION}

In this paper, an effective homotopy-based inverse design method is used to optimized the heating flux distribution around the 2D profile of the leading edge in the hypersonic regime. Numerical examples show that the optimization is successful. The peak value of the heating flux decreases about 4.6\%. Although the cases presented in this paper aims at 2D shapes, the inverse design method can be easily extend to 3D configurations. However, the computer time will increase inevitably. Besides, the results of this paper shoule be validated by wind tunnel experiment to further assurance.

\section{Acknowledgements}

The support of the National Natural Science Foundation of China (90916013) is gratefully acknowledged.

\section{REFERENCES}

1. Anderson J D Jr. Hypersonic and High Temperature Gas Dynamics. McGraw-Hill. New York (1989)

2. Yegorov I V, Yegorova M V, Ivanov D V, et al. Numerical study of hypersonic viscous flow about plates located behind a cylinder, AIAA-97-2573, 1997

3. Wieting AR. Experimental study of shock wave interference heating on a cylindrical leading edge. NASA-TM100484. 1987

4. Santos WF N. Leading-edge bluntness effects on aerodynamic heating and drag of power law body in low-density hypersonic flow. J. Braz. Soc. Mech. Sci. \& Eng. 2005; 27(3): 236-242

5. Sidra IS, Goldsteiny DB. Numerical and experimental investigation of the reduction of hypersonic nose tip ablation. AIAA-1998-0169 1998

6. DeJarnette FR, Hamilton HH, Weilmuenster KJ, et al. A Review of some approximate methods used in aerodynamic heating analysis. J. of Thermophysics and Heat Transfer. 1987; 1: 5-12

7. Dulikravich GS. Design and Optimization Tools Development. Chapters 10-15 in New Design Concepts for High Speed Air Transport. (H. Sobieczkyedn edn.) Springer. Wien/New York (1997)

8. Cui K, Yang GW, A continuation method of parameter inversion for non-equilibrium convection-dispersion equation. Chinese Physics Letters. 2005; 22(11): 2738-2741 